\title{
Barreiras percebidas para a prática de atividade física entre universitários de Educação Física
}

\author{
Perceived barriers to physical activity in Physical Education students
}

\author{
André de Araújo Pinto', Gaia Salvador Claumann', Paloma Cidade Cordeiro², Erico Pereira Gomes Felden', Andreia Pelegrini
}

\section{Resumo}

O objetivo deste estudo foi identificar as barreiras percebidas para a prática de atividade física entre universitários de Educação Física de Florianópolis, e testar a associação dessas barreiras percebidas com o nível de atividade física entre os cursos de Bacharelado e Licenciatura em Educação Física. Participaram 301 universitários (173 homens e 128 mulheres) do curso de Educação Física (161 da Licenciatura e 140 do Bacharelado), com média de idade de 23,1(4,9) anos. Foram coletadas informações sociodemográficas, prática de atividade física (Estágios de Mudança de Comportamento relacionados à Atividade Física) e barreiras percebidas. As barreiras foram analisadas individualmente e agrupadas em domínios (ambiental, comportamental, físico e social). Associações entre barreiras percebidas (por domínios) e prática de atividades físicas foram testadas por meio de Regressão de Poisson. As principais barreiras percebidas pelos universitários, de ambos os cursos, foram jornada de trabalho extensa $($ Bacharelado $=16,5 \%$, Licenciatura $=30,4 \%)$, falta de energia $($ Bacharelado $=11,5 \%$, Licenciatura $=10,6 \%)$ e falta de companhia (Bacharelado $=7,2 \%$, Licenciatura $=8,9 \%)$. As barreiras do domínio social estiveram associadas à inatividade física no Bacharelado $(\mathrm{RP}=3,17$; IC95\%= 1,52-6,60) e na Licenciatura $(\mathrm{RP}=1,89$; $\mathrm{IC} 95 \%=1,14-3,13)$. Conclui-se que as barreiras mais prevalentes foram jornada de trabalho extensa, falta de energia e falta de companhia. Quando agrupadas por domínios, as sociais, em ambos os cursos, estiveram associadas à inatividade física. Ações devem ser voltadas a esses aspectos para diminuir seu impacto na tomada de decisão sobre a prática de atividades físicas.

\section{Palavras-chave}

Atividade física; Estudantes; Estilo de vida; Comportamentos saudáveis.

\begin{abstract}
This study aimed to identify the perceived barriers for physical activity among Physical Education college students of Florianopolis, and to test the association of these perceived barriers with physical activity level among Bachelor and Licenciate courses of Physical Education. Participants were 301 (173 male and 128 female) Physical Education college students (161 of Licenciate and 140 of Bachelor), with mean age of 23.1(4.9) years. Sociodemographic information, physical activity (Stages of Behavior Change for Physical Activity) and perceived barriers were collected. Barriers were analyzed individually and grouped in domains (environmental, behavioral, physical and social). Associations between perceived barriers and physical activity were tested by means of Poisson Regression. The main perceived barriers by students, of both courses, were extensive working time (Bachelor $=16.5 \%, \mathrm{Li}$ cenciate $=30.4 \%)$, lack of energy (Bachelor $=11.5 \%$, Licenciate $=$ $10.6 \%$ ) and lack of company (Bachelor $=7.2 \%$, Licenciate $=8.9 \%$ ). Barriers of social domain were associated to physical inactivity in Bachelor $(P R=3.17 ; C I 95 \%=1.52-6.60)$ and in Licenciate $(P R=$ 1.89; $C 195 \%=1.14-3.13)$. We conclude that the most prevalente perceived barriers were extensive working time, lack of energy and lack of company. When grouped by domains, social barriers, for both courses, were associated to physical inactivity. Actions should be directed to these aspects to reduce its impact in decision taking to physical activity.
\end{abstract}

\section{Keywords}

Physical activity; Students; Life style; Health behavior.

\section{Introdução}

Os benefícios da prática regular de atividade física (AF) para a saúde estão bem documentados, destacan-

1 Universidade do Estado de Santa Catarina. Programa de Pós-graduação em Ciências do Movimento Humano. Florianópolis, Santa Catarina, Brasil.

2 Universidade do Estado de Santa Catarina. Departamento de Educação Física. Florianópolis, Santa Catarina, Brasil. do-se seus efeitos positivos em aspectos físicos, sociais e mentais ${ }^{1,2}$ que, juntos, compreendem o conceito de saúde ${ }^{3}$. Por outro lado, evidências sugerem que níveis insuficientes de AF estão associados ao aumento do risco de desenvolvimento de diversas doenças ${ }^{4}$, fazendo com que a inatividade física esteja entre as 10 principais causas de mortalidade ${ }^{5}$. Essas evidências são 
preocupantes, principalmente nos dias atuais, em que aproximadamente $1 / 3 \mathrm{da}$ população adulta mundial não atende as recomendações necessárias de $\mathrm{AF}^{6}$.

Dentre a população adulta exposta à níveis insuficientes de AF, destacam-se os universitários, pois, apesar de terem acesso facilitado aos conhecimentos sobre a saúde durante o período acadêmico, por meio do acesso às informações atualizadas, leituras científicas cotidianas e internet, ainda são elevadas as prevalências deste comportamento nesta população, tanto a nível nacional quanto internacional ${ }^{7,8}$.

Diante disso, estudos nacionais ${ }^{9,10} \mathrm{e}$ internacionais ${ }^{11-13}$ conduzidos em universitários de diferentes áreas de conhecimento, buscaram identificar as barreiras mais prevalentes para a prática de AF nessa população, pois, a rotina do ensino superior exige maiores responsabilidades acadêmicas e, muitas vezes, ocupacionais, de forma que os cuidados necessários para a saúde, do ponto de vista da AF, podem ficar em segundo plano.

Destaca-se que, no Brasil, as informações sobre as barreiras para a prática de $\mathrm{AF}$ são provenientes de inquéritos conduzidos, em sua maioria, com universitários dos cursos de Educação Física ${ }^{14,15}$. Especificamente nessa população, no Paraná ${ }^{\text {e }}$ na $\mathrm{Bahia}^{15}$, os universitários apontaram como principais barreiras para a prática de $\mathrm{AF}$ as jornadas de trabalho e estudo extensas ${ }^{9,15}$, as obrigações familiares e o clima desconfortável ${ }^{15}$.

Contudo, não foi observado na literatura pesquisada se as barreiras percebidas diferem entre os tipos de habilitação em Educação Física (Bacharelado e Licenciatura), sendo importante considerar a análise estratificada para cada habilitação, pois as mesmas apresentam atividades curriculares e áreas de atuação distintas. Ademais, apesar de se esperar que os universitários dos cursos de Educação Física sejam suficientemente ativos, essa hipótese pode não ser verdadeira, principalmente em relação às AF realizadas fora do contexto universitário (disciplinas com aulas práticas e estágios obrigatórios).

Dessa forma, a identificação das barreiras para a prática de AF nessa população é de grande relevância, pois pode possibilitar o direcionamento de estratégias mais objetivas, que vão além das obrigações com as práticas acadêmicas, para que esse público encare a $\mathrm{AF}$ regular como necessidade e não como obrigação, mas que deve se perpetuar além da universidade. Assim, o presente estudo teve como objetivo principal identificar as barreiras percebidas para a prática de AF entre universitários de Educação Física de Florianópolis. Ainda, o objetivo secundário foi testar a associação dessas barreiras percebidas com o nível de AF entre os cursos de Bacharelado e Licenciatura em Educação Física.

\section{Métodos}

Trata-se de um estudo transversal conduzido no segundo semestre de 2013. O mesmo está vinculado ao projeto "Estudo longitudinal da saúde e cognição de acadêmicos de Educação Física da UDESC", aprovado pelo Comitê de Ética em Pesquisa com Seres Humanos da Universidade do Estado de Santa Catarina (parecer $n^{\circ} 83192 / 2012$ ).

Constituíram a população do estudo os universitários dos cursos de Educação Física (com habilitações em Bacharelado e Licenciatura) de uma universidade pública da cidade Florianópolis. Conforme informações da direção de ensino da instituição, no período de realização do estudo haviam 500 universitários regularmente matriculados nas oito fases dos cursos de graduação. Para a representatividade amostral, seria necessária a participação de 274 indivíduos, considerando-se um erro de quatro pontos percentuais e prevalência de $50 \%{ }^{16}$. Com intuito de ame- 
nizar eventuais perdas e/ou recusas, acrescentou-se mais $10 \%$, o que resultou em amostra mínima de 301 universitários. Os universitários presentes em sala de aula no momento da coleta de dados, que aceitaram participar livremente e assinaram o Termo de Consentimento Livre e Esclarecido participaram do presente estudo, totalizando 301 indivíduos, que representam 60,2\% da população alvo.

As coletas de dados ocorreram no período de aula, nas dependências da universidade, em dias e horários agendados com um dos professores que ministravam aulas para cada fase do curso. A equipe de coleta de dados explicou aos universitários presentes os objetivos e a importância do estudo e convidou a todos para responderem aos questionários, compostos por questões sociodemográficas, sobre a prática de $\mathrm{AF}$ e as barreiras percebidas para a mesma.

A respeito das questões sociodemográficas, os participantes informaram o seu sexo (masculino, feminino), idade (em anos completos), estado civil (com companheiro, sem companheiro) e turno de estudo (diurno, em que estudam os universitários do curso de Bacharelado, e noturno, em que estudam os universitários da Licenciatura). Responderam também sobre sua ocupação por meio da seguinte questão: "Você trabalha atualmente?". As alternativas para resposta foram: a) Não trabalho; b) Faço estágio profissionalizante (com ou sem remuneração); c) Trabalbo com salário ed) Trabalho sem salário. Para fins de análise, optou-se por agrupar os universitários em duas categorias: trabalhando (categorias b, c e d) e desempregado (categoria a). Aqueles que trabalhavam deveriam indicar a carga horária de trabalho semanal (< 20 horas e $\geq 20$ horas). O nível econômico foi estimado pelo questionário da Associação Brasileira de Empresas de Pesquisa ${ }^{17}$ vigente à época, que classifica a população brasileira, de acordo com seu poder de compra, nos estratos econômicos, em ordem decrescente: A1, A2, B1, B2, C1, C2, D e E. Nenhum dos universitários do presente estudo foi classificado no estrato E, e apenas cinco foram classificados no estrato D. A partir disso, os demais estratos econômicos foram agrupados e considerados de nível alto (estratos A1, A2 e B1) e médio/baixo (B2, C1, C2 e D), conforme classificação utilizada em estudo prévio $^{18}$.

Para classificar os universitários quanto à prática de AF foi empregado o questionário que retrata os estágios de mudança de comportamento relacionados à $\mathrm{AF}$ (EMCAF), validado para adultos jovens ${ }^{19}$. O questionário dispõe de perguntas relacionadas à $\mathrm{AF}$, com respostas "sim" ou "não" e obedece a seguinte sequência: pré-contemplação (sujeito não anseia modificar seu comportamento num futuro próximo), contemplação (existe o intuito de mudar o comportamento, mas não imediatamente), preparação (existe o intuito de mudar o comportamento nos próximos seis meses), ação (mudanças recentes no comportamento) e manutenção (as mudanças no comportamento já perduram por mais de seis meses). Para fins de análise, os EMCAF foram reagrupados em duas categorias: ativos (ação e manutenção) e inativos fisicamente (pré-contemplação, contemplação e preparação), conforme categorização utilizada previamente $e^{9,20-22}$. Foi solicitado aos universitários que considerassem somente as $\mathrm{AF}$ realizadas fora da universidade (sem vínculos acadêmicos, como aulas práticas das disciplinas e atividades realizadas em estágios obrigatórios).

As barreiras percebidas para a prática de $\mathrm{AF}$ foram identificadas por meio de um questionário proposto para indivíduos adultos e validado previamente por Martins e Petroski ${ }^{23}$. No instrumento são apresentados 19 motivos que possivelmente bloqueiam ou impedem a realização de AF, cujas respostas são obtidas por meio de escala likert $(1=$ nunca; $2=$ raramente; 3 às vezes; $4=$ quase sempre e $5=$ sempre). Considerou-se como barreira percebida os valores correspondentes as 
respostas " 4 e 5 ” e barreira ausente os valores “ 1,2 e 3”24,25. A prevalência das 19 barreiras foi analisada e, posteriormente, as mesmas foram agrupadas e analisadas em quatro domínios principais: barreiras ambientais, sociais, comportamentais e físicas, conforme categorização utilizada em estudo prévio ${ }^{26}$.

Os dados foram analisados inicialmente por meio de estatística descritiva, a partir da distribuição de frequências (absoluta e relativa). Posteriormente foram empregados os testes Qui-quadrado e/ou Exato de Fischer para comparar se havia diferença da AF praticada entre os cursos (Bacharelado e Licenciatura) e para identificar possíveis associações entre as variáveis. Utilizou-se a regressão de Poisson para verificar possíveis associações entre os domínios das barreiras para a prática de AF e a AF estimada. Foram conduzidas análises bruta e ajustada estratificadas pela habilitação do curso (Bacharelado e Licenciatura). Na análise ajustada foram incluídos todos os domínios das barreiras, além do sexo, idade e nível econômico dos participantes. Todas as análises foram realizadas no programa SPSS, versão 20.0, e o nível de significância adotado foi de 5\%.

\section{Resultados}

Os universitários participantes do presente estudo apresentaram média de idade de 23,1(4,9) anos. Na Tabela 1 são apresentadas as características gerais da amostra total e estratificado por curso. A maior parte da amostra foi constituída por indivíduos do sexo masculino (57,5\%), com idades de 20 a 30 anos (56,1\%), que estavam sem companheiro $(64,1 \%)$, trabalhavam $(76,0 \%)$, com carga horária de trabalho semanal $\geq 20$ horas $(55,0 \%)$ e apresentavam nível econômico médio/baixo $(53,8 \%)$.

TABELA 1 - Caracterização dos universitários do curso de Educação Física (habilitações em Bacharelado e Licenciatura). Florianópolis, 2013.

\begin{tabular}{|c|c|c|c|c|c|c|}
\hline \multirow{2}{*}{ Variáveis } & \multicolumn{2}{|c|}{ Total } & \multicolumn{2}{|c|}{ Bacharelado } & \multicolumn{2}{|c|}{ Licenciatura } \\
\hline & $n$ & $\%$ & $n$ & $\%$ & $n$ & $\%$ \\
\hline \multicolumn{7}{|l|}{ Sexo } \\
\hline Masculino & 173 & 57,5 & 84 & 60,0 & 89 & 55,3 \\
\hline Feminino & 128 & 42,5 & 56 & 40,0 & 72 & 44,7 \\
\hline \multicolumn{7}{|l|}{ Idade } \\
\hline$\leq 20$ anos & 103 & 34,8 & 55 & 40,4 & 48 & 30,0 \\
\hline 20 a 30 anos & 166 & 56,1 & 74 & 54,4 & 92 & 57,5 \\
\hline$\geq 30$ anos & 27 & 9,1 & 7 & 5,2 & 20 & 12,5 \\
\hline \multicolumn{7}{|l|}{ Estado civil } \\
\hline Com companheiro(a) & 106 & 35,9 & 46 & 33,3 & 60 & 38,2 \\
\hline Sem companheiro(a) & 189 & 64,1 & 92 & 66,7 & 97 & 61,8 \\
\hline \multicolumn{7}{|l|}{ Ocupação } \\
\hline Desempregado(a) & 72 & 24,0 & 41 & 29,3 & 31 & 19,3 \\
\hline Trabalhando & 228 & 76,0 & 99 & 70,7 & 130 & 80,7 \\
\hline \multicolumn{7}{|l|}{ Carga horária semanal } \\
\hline$<20$ horas & 135 & 45,0 & 75 & 53,6 & 60 & 37,5 \\
\hline$\geq 20$ horas & 165 & 55,0 & 65 & 46,4 & 100 & 62,5 \\
\hline \multicolumn{7}{|l|}{ Nível econômico } \\
\hline Alto & 132 & 46,2 & 68 & 51,1 & 64 & 41,8 \\
\hline Médio/Baixo & 154 & 53,8 & 65 & 48,9 & 89 & 58,2 \\
\hline
\end{tabular}

n: frequência absoluta; \%: frequência relativa.

Observou-se que a prevalência de inatividade física entre os universitários foi de 38,5\%, sendo superior naqueles do curso de Licenciatura (46,0\%) em relação aos do Bacharelado (30,0\%) ( $\mathrm{p}=0,005)$ (Figura 1). 


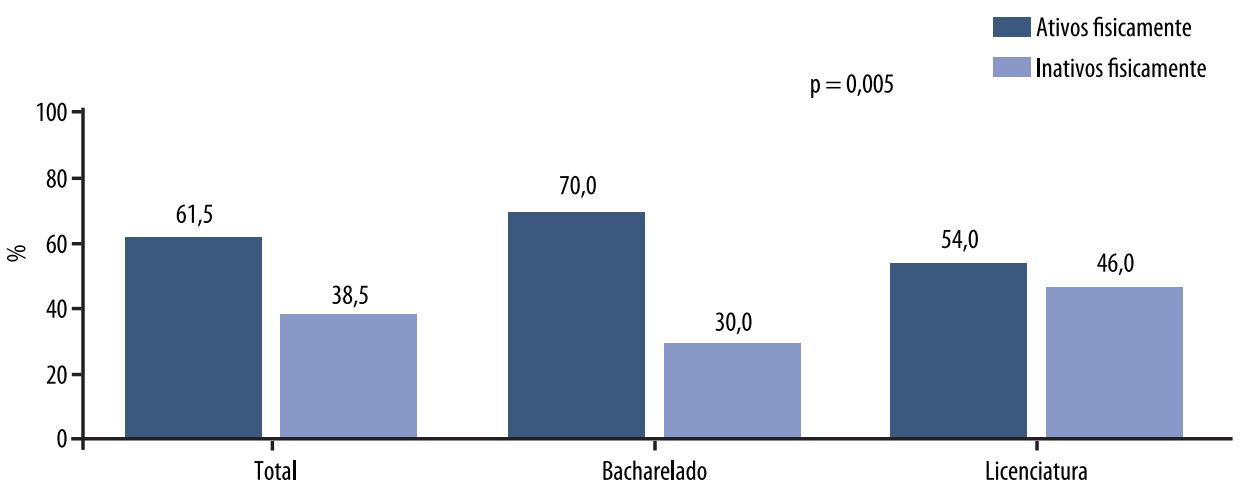

FIGURA 1 - Atividade física em universitários de Educação Física de acordo com a habilitação do curso. Florianópolis, 2013.

As barreiras percebidas com maior frequência pelos universitários de ambos os cursos (Bacharelado e Licenciatura) foram jornada de trabalho extensa (16,5\% e $30,4 \%$, respectivamente), falta de energia (11,5\% e 10,6\%, respectivamente) e falta de companhia $(7,2 \%$ e $8,9 \%)$. Houve associação entre a jornada de trabalho extensa e a habilitação do curso $(\mathrm{p}=0,005)$ (Tabela 2$)$.

TABELA 2 - Barreiras percebidas para a prática de atividades físicas em universitários do curso Educação Física (habilitações em Bacharelado e Licenciatura). Florianópolis, 2013.

\begin{tabular}{|c|c|c|c|c|c|}
\hline \multirow{3}{*}{ Barreiras } & \multicolumn{4}{|c|}{ Curso } & \multirow{3}{*}{ p-valor } \\
\hline & \multicolumn{2}{|c|}{ Bacharelado } & \multicolumn{2}{|c|}{ Licenciatura } & \\
\hline & $n$ & $\%$ & $\mathrm{n}$ & $\%$ & \\
\hline \multicolumn{6}{|l|}{ Domínio ambiental } \\
\hline Falta de clima adequado* & 6 & 4,3 & 7 & 4,4 & 0,971 \\
\hline Falta de espaço disponível para a prática** & 4 & 2,9 & 10 & 6,2 & 0,169 \\
\hline Falta de equipamento disponível** & 3 & 2,2 & 8 & 5,0 & 0,197 \\
\hline Ambiente insuficientemente seguro** & 3 & 2,2 & 4 & 2,5 & 0,845 \\
\hline \multicolumn{6}{|l|}{ Domínio social } \\
\hline Jornada de trabalho extensa* & 23 & 16,5 & 49 & 30,4 & 0,005 \\
\hline Compromissos familiares* & 6 & 4,3 & 11 & 7,0 & 0,327 \\
\hline Tarefas domésticas** & 3 & 2,2 & 9 & 5,7 & 0,128 \\
\hline Falta de companhia** & 10 & 7,2 & 14 & 8,9 & 0,600 \\
\hline Falta de incentivo de familiares e/ou amigos** & 3 & 2,2 & 6 & 3,8 & 0,422 \\
\hline Falta de recursos financeiros* & 7 & 5,0 & 15 & 9,4 & 0,147 \\
\hline Falta de conhecimento ou orientação sobre $\mathrm{AF}^{* *}$ & 3 & 2,2 & 3 & 1,9 & 0,862 \\
\hline \multicolumn{6}{|l|}{ Domínio comportamental } \\
\hline Mau humor** & 1 & 0,7 & 4 & 2,5 & 0,231 \\
\hline Medo de lesionar-se** & 2 & 1,4 & 1 & 0,6 & 0,481 \\
\hline Preocupação com a aparência durante a prática** & 1 & 0,7 & 3 & 1,9 & 0,386 \\
\hline Falta de interesse em praticar** & 4 & 2,9 & 3 & 1,9 & 0,567 \\
\hline \multicolumn{6}{|l|}{ Domínio físico } \\
\hline Limitações físicas** & 2 & 1,4 & 6 & 3,8 & 0,217 \\
\hline Dores leves ou mal estar* & 4 & 2,9 & 3 & 1,9 & 0,567 \\
\hline Falta de energia** & 16 & 11,5 & 17 & 10,6 & 0,807 \\
\hline Falta de habilidade física** & 2 & 1,4 & 1 & 0,6 & 0,485 \\
\hline
\end{tabular}

n: frequência absoluta; \%; frequência relativa. * Qui-quadrado. ** Exato de Fischer. 
Nas Tabelas 3 e 4 são apresentadas as análises de associação entre as barreiras percebidas para a prática de AF e inatividade física nos universitários do curso de Bacharelado (Tabela 3) e Licenciatura (Tabela 4). Após ajuste por todas as barreiras, sexo, idade e nível econômico, as barreiras sociais estiveram associadas à inatividade física em ambos os cursos. Os universitários do curso de Bacharelado e de Licenciatura que percebiam barreiras sociais para a prática de $\mathrm{AF}$ apresentaram, probabilidade maior $(\mathrm{RP}=3,17 ; \mathrm{IC} 95 \%=1,52-6,60 ; \mathrm{RP}=1,89 ; \mathrm{IC} 95 \%=1,14-3,13$, respectivamente $)$ de serem inativos fisicamente quando comparados aos seus pares que não percebiam.

TABELA 3 - Prevalência e razões de prevalência de inatividade física, de acordo com as barreiras percebidas em universitários da habilitação em Bacharelado do curso de Educação Física. Florianópolis, 2013.

\begin{tabular}{|c|c|c|c|}
\hline \multirow{2}{*}{ Variáveis } & Inatividade física & Análise bruta & Análise ajustada* \\
\hline & $n(\%)$ & RP (IC95\%) & RP (IC95\%) \\
\hline \multicolumn{4}{|l|}{ Ambientais } \\
\hline Ausência & $34(27,7)$ & 1,00 & 1,00 \\
\hline Presença & $8(57,1)$ & $2,06(1,23-3,59)$ & $1,20(0,49-2,92)$ \\
\hline \multicolumn{4}{|c|}{ Comportamentais } \\
\hline Ausência & $37(28,0)$ & 1,00 & 1,00 \\
\hline Presença & $5(71,4)$ & $2,55(1,48-4,38)$ & $1,58(0,54-4,61)$ \\
\hline \multicolumn{4}{|l|}{ Físicas } \\
\hline Ausência & $31(26,1)$ & 1,00 & 1,00 \\
\hline Presença & $11(55,0)$ & $2,11(1,28-3,48)$ & $1,05(0,44-2,50)$ \\
\hline \multicolumn{4}{|l|}{ Sociais } \\
\hline Ausência & $19(19,0)$ & 1,00 & 1,00 \\
\hline Presença & $23(57,5)$ & $3,03(1,86-4,91)$ & $3,17(1,52-6,60)$ \\
\hline
\end{tabular}

n: frequência absoluta; \%: frequência relativa; RP: razão de prevalência; IC95\%: intervalo de confiança de $95 \% .{ }^{*}$ Ajustada por todas as barreiras mais o sexo, idade e nível econômico.

TABELA 4 - Prevalência e razões de prevalência inatividade física de acordo com as barreiras percebidas em universitários da habilitação em Licenciatura do curso de Educação Física. Florianópolis, 2013.

\begin{tabular}{|c|c|c|c|}
\hline \multirow{2}{*}{ Variáveis } & Inatividade física & Análise bruta & Análise ajustada* \\
\hline & $n(\%)$ & RP (IC95\%) & RP (IC95\%) \\
\hline \multicolumn{4}{|l|}{ Ambientais } \\
\hline Ausência & $61(43,6)$ & 1,00 & 1,00 \\
\hline Presença & $12(60,0)$ & $1,38(0,92-2,06)$ & $1,06(0,53-2,11)$ \\
\hline \multicolumn{4}{|l|}{ Comportamentais } \\
\hline Ausência & $65(43,0)$ & 1,00 & 1,00 \\
\hline Presença & $8(88,9)$ & $2,07(1,54-2,77)$ & $1,36(0,62-2,97)$ \\
\hline \multicolumn{4}{|l|}{ Físicas } \\
\hline Ausência & $57(40,7)$ & 1,00 & 1,00 \\
\hline Presença & $16(80,0)$ & $1,97(1,46-2,64)$ & $1,50(0,81-2,75)$ \\
\hline \multicolumn{4}{|l|}{ Sociais } \\
\hline Ausência & $30(31,9)$ & 1,00 & 1,00 \\
\hline Presença & $44(65,7)$ & $2,06(1,46-2,90)$ & $1,89(1,14-3,13)$ \\
\hline
\end{tabular}

n: frequência absoluta; \%: frequência relativa; RP: razão de prevalência; IC95\%: intervalo de confiança de $95 \%$. *Ajustada por todas as barreiras mais o sexo, idade e nível econômico.

\section{Discussão}

As barreiras percebidas para a prática de $\mathrm{AF}$ mais frequentes entre os universitários de Educação Física participantes do presente estudo (de ambas as habilitações, Bacharelado e Licenciatura) foram, em ordem decrescente, a jornada de trabalho 
extensa, falta de energia e falta de companhia. As análises de associação revelaram que aqueles que percebiam barreiras sociais (para universitários de ambas as habilitações) apresentaram maior probabilidade de serem inativos fisicamente.

Em relação às barreiras mais prevalentes, resultados semelhantes foram encontrados em estudos prévios ${ }^{9,10}$ conduzidos com a população universitária, principalmente em relação à jornada de trabalho. No estudo de Rigoni et al. ${ }^{9}$, os universitários de Educação Física apontaram a jornada de trabalho extensa, além da jornada de estudo extensa, seguidas da falta de companhia, como barreiras mais frequentes para a prática de AF. Resultado que também foi semelhante em estudo com universitários de diferentes cursos de uma universidade pública da $\mathrm{Bahia}^{10} \mathrm{e}$ de outros países como o Egito ${ }^{11} \mathrm{e}$ Espanha ${ }^{13}$, os quais também foram conduzidos em universidades públicas.

Ressalta-se que apesar da jornada de trabalho extensa ter sido a principal barreira para a prática de AF apontada pelos universitários de ambos os cursos, é possível que para aqueles da Licenciatura essa barreira tenha um impacto maior sobre suas decisões para a realização ou não de AF. Isto porque as aulas do curso de Licenciatura ocorrem no período noturno na instituição pesquisada, diferente das aulas do Bacharelado que acontecem no período matutino. Dessa forma, os estudantes da Licenciatura possuem mais horas livres para o trabalho e, de fato, 62,5\% tem carga horária de trabalho superior a 20 horas semanais. Além disso, os universitários dessa habilitação são em sua maioria mais velhos e possuem companheiros, o que sugere que podem ser responsáveis pela manutenção das condições de sobrevivência de suas famílias, tendo menos tempo e talvez disposição para praticar AF.

Em linhas gerais, esses achados significam a necessidade de administração do tempo dos universitários não somente dos cursos de Educação Física ${ }^{9}$, mas também de outros cursos ${ }^{11,13}$ para que a AF seja encarada como uma prioridade e incrementada ao cotidiano ${ }^{9}$. A respeito do presente estudo, compreende-se a dimensão do exercício das atividades ocupacionais dessa população, pois muitos vêm de outros municípios vizinhos, encaram longas horas de deslocamento com transporte coletivo, e ainda precisam trabalhar para manter seu sustento e por isso se encontram no contexto da universidade pública. Tais circunstâncias podem justificar o não engajamento dos universitários em AF, especialmente se somadas às atividades acadêmicas, levando-os a perceberem o dia com duração mais curta e a optarem por ficar em casa realizando atividades que não envolvam esforço físico e que ofereçam maior sensação de prazer e descanso.

Assim como observado no presente estudo, a falta de energia também tem sido apontada como uma das barreiras mais percebidas para a prática de AF por universitários ${ }^{9,12}$, e o cansaço físico por indivíduos adultos (com idade superior a 20 anos $)^{27}$. Mauro et al. ${ }^{28}$ sugerem que o cansaço físico pode, muitas vezes, refletir em uma percepção de dores físicas, tornando-se, portanto, um fator de impedimento para a realização de atividades físicas. Com base nesse raciocínio, parece fazer sentido que a jornada de trabalho extensa, barreira mais citada pelos universitários do presente estudo, reflita na percepção da falta de energia (que pode estar relacionada não somente ao tipo de atividade realizada no trabalho, mas também ao tempo envolvido e às posições adotadas, como estar sentado por longo período de tempo). Isso pode gerar sensação de cansaço físico e dores físicas levando os universitários a procurar o descanso em seu tempo livre.

A falta de companhia foi a terceira barreira mais prevalente para os universitários de Educação Física. Uma revisão sistemática concluiu que os colegas e amigos desempenham um papel importante nos níveis de AF de adolescentes, pois todos 
os estudos incluídos encontraram associação direta entre essas variáveis, por meio principalmente do suporte social ou da presença dos colegas e amigos durante a $\mathrm{AF}^{29}$. A mesma relação também já foi identificada em indivíduos adultos ${ }^{30}$. Dessa forma, a companhia de outras pessoas parece ser um fator essencial para a prática de AF independentemente da faixa etária investigada, e, na universidade por ser um contexto em que se encontram pessoas de diferentes idades parece plausível a hipótese de que esse fator também se faça presente.

A respeito das análises de associação, verificou-se que os universitários, de ambas as habilitações, que percebiam barreiras sociais para a prática de AF apresentaram maior probabilidade de serem inativos fisicamente. É importante destacar que o domínio social inclui fatores relacionados a "jornada de trabalho extensa" e a "falta de companhia", os quais foram os mais apontados como barreiras. Dessa forma, parece adequado afirmar que esses indivíduos não somente percebem esses fatores como obstáculos, mas que de fato, os mesmos os impossibilitam de iniciar ou manter a prática de $\mathrm{AF}$, ao menos no lazer, por exemplo, pois é possível que sejam ativos no contexto do trabalho. Nesse caso, seria importante dispor de maiores informações a respeito do tipo de atividade realizada pelos universitários no trabalho, o que possibilitaria uma compreensão mais aprofundada desta situação.

Diante disso, na hipótese de os universitários não atenderem as recomendações de AF, considerando todos os seus domínios (lazer, deslocamento, trabalho e doméstico), julga-se necessário que existam iniciativas no ambiente de trabalho que contribuam para uma rotina mais ativa (ou que envolvam atividades de alongamento e relaxamento muscular para os casos de trabalhos que exijam elevado esforço físico), visto que se não houver tempo disponível para a prática de AF além do horário de trabalho, ao menos ela é oportunizada, de maneira apropriada, nesse contexto. Ademais, em relação à falta de companhia, é fundamental que as pessoas tenham autonomia para a AF, para que sejam capazes de realizá-la mesmo na ausência de parceiros para a prática.

Os resultados do presente estudo agregam informações importantes à literatura existente sobre a temática, por abordar uma quantidade expressiva de universitários, de diferentes períodos, do curso de Educação Física e por conduzir análises separadas para as habilitações em Bacharelado e Licenciatura, que podem apresentar características distintas devido ao turno de estudo de cada habilitação (matutino e noturno, respectivamente), às diferentes disciplinas e cargas horárias das mesmas bem como diferentes ênfases na área de atuação. Apesar disso, os resultados encontrados foram semelhantes em ambas as habilitações e corroboram o corpo de evidências sobre a temática na população universitária ${ }^{9,11-13}$.

Destaca-se ainda que algumas limitações devem ser consideradas para a interpretação dos dados apresentados. Por exemplo, o delineamento transversal empregado não permite inferir causalidade entre as variáveis investigadas; a amostra investigada foi constituída por um grupo de indivíduos mais suscetíveis ao envolvimento em práticas habituais de atividades físicas e esportivas, dificultando a extrapolação dos resultados para estudantes de outros cursos universitários. Outra questão é que não foram realizadas análises estratificadas por sexo, que apesar de ter sido utilizado como ajuste pode ter limitado os achados. Além disso, o instrumento utilizado para a avaliação da prática de $\mathrm{AF}$ (EMCAF) não foi proposto para essa finalidade e pode não refletir o verdadeiro nível de AF dos universitários, entretanto, tem sido utilizado dessa maneira por estudos prévios, 20-22.

Sugere-se, para estudos futuros que desejam investigar as barreiras para a prática de AF na população universitária, que além da jornada de trabalho extensa, 
fator apontado como barreira mais frequentemente na presente investigação, incluam a rotina de estudos como possível barreira percebida. Isto porque muitos universitários não trabalham, porém, a rotina de estudos por si só, que envolve o período dedicado às atividades em sala de aula além do tempo despendido para estudos em outros momentos, atividades extracurriculares de pesquisa e extensão, já é o suficiente para impedir a prática de AF. Além disso, em relação aos indivíduos que trabalham, conhecer o tipo de atividade realizada nesse contexto pode auxiliar na compreensão mais aprofundada da relação entre AF e trabalho. Ademais, sugere-se a realização de análises futuras estratificadas por sexo, com intuito de reduzir o possível viés de confusão.

Conclui-se que as principais barreiras para a prática de AF percebidas pelos universitários das habilitações em Bacharelado e Licenciatura do curso de Educação Física foram, em ordem decrescente, a jornada de trabalho extensa, a falta de energia e a falta de companhia. As barreiras do domínio social estiveram associadas à inatividade física, para ambas as habilitações.

Frente a esses achados, recomenda-se o desenvolvimento de políticas universitárias tais como instalações de moradia gratuitas para os universitários nas próprias universidades, auxílio de bolsas de estudos para que facilite o custeio dos universitários que moram mais distantes da faculdade, e ainda oferta de práticas de AF gratuitas, incluindo as esportivas no contraturno em que os universitários estudam, por meio de ações de extensão, por exemplo.

\section{Contribuiç̧ão dos autores}

A. A. Pinto participou da análise de dados e redação do artigo. G. S. Claumann e P. C. Cordeiro participaram da concepção do projeto, coleta de dados e análise crítica. E. P. G. Felden e A. Pelegrini idealizaram o projeto e participaram da revisão crítica intelectual do artigo. Todos os autores aprovaram a versão final a ser publicada.

\section{Agradecimentos}

À Fundação de Amparo à Pesquisa do Amazonas (FAPEAM) e a Universidade do Estado de Santa Catarina (UDESC), pelas respectivas bolsas de estudos.

\section{Referências}

1. Bastos JP, Araújo CLP, Hallal PC. Prevalence of insufficient physical activity and associated factors in Brazilian adolescents. J Phys Act Health. 2008; 5(6): 777-94.

2. Strong WB, Malina RM, Blimkie CJ, Daniels SR, Dishman RK, Gutin B, et al. Evidence based physical activity for school-age youth. J Pediatr. 2005; 146(6): 732-7.

3. World Health Organization. International Society of Hypertension Guidelines for the Management of Hypertension. J Hypertens. 1999; 17: 151-83.

4. World Health Organization. Preventing Chronic Diseases: a vital investment: WHO global report. WHO; 2005.

5. World Health Organization. Global recommendations on physical activity for health. Geneva: WHO; 2010.

6. Hallal PC, Andersen LB, Bull FC, Guthold R, Haskell W, Ekelund U. Global physical activity levels: surveillance progress, pitfalls, and prospects. Lancet. 2012; 380(9838): 247-57.

7. Sousa TF. Inatividade física em universitários brasileiros: uma revisão sistemática. Rev Atenção Saúde. 2011; 9(29): 47-55.

8. Irwin J. Prevalence of university students' sufficient physical activity: a systematic review. Percept Mot Skills. 2004; 98(3): 927-43.

9. Rigoni PAG, Nascimento Junior JRA, Costa GNFS, Vieira LF. Estágios de mudança de comportamento e percepção de barreiras para a prática de atividade física em 
universitários do curso de Educação Física. Rev Bras Ativ Fís Saú. 2012; 17(2): 87-92.

10. Sousa TF, Fonseca AS, Barbosa AR. Perceived barriers by university students in relation the leisure-time physical activity. Rev Bras Cineantropom Desempenho Hum. 2013; 15(2): 164-73.

11. El-gilany A-H, Badawi K, El-khawaga G, Awadalla N. Physical activity profile of students in Mansoura University, Egypt. EMHJ. 2011; 17(8): 694-702.

12. Daskapan A, Tuzun EH, Eker L. Perceived barriers to physical activity in university students. J Sports Sci Med. 2006; 5(4): 615-20.

13. Gomes-Lopez M, Gallegos AG, Extremera AB. Perceived barriers by university students in the practice of physical activities. J Sports Sci Med. 2010; 9(3): 374-81.

14. Oliveira CS, Gordia AP, Quadros TMB, Campos W. Atividade física de universitários brasileiros: uma revisão da literatura. Rev Atenção Saúde. 2014; 12(42): 71-7.

15. Sousa TF, Santos SFS, José HPM. Barreiras percebidas à prática de atividade física no Nordeste do Brasil. Pensar a Prática. 2010; 13(1): 1-15.

16. Rodrigues PC. Bioestatística. Niterói: Editora da Universidade Federal Fluminense, 2002.

17. Associação Brasileira de Empresas de Pesquisa. Critério de Classificação Econômica Brasil 2010. ABEP; 2010.

18. Claumann GS, Pereira EF, Pelegrini A. Prática de caminhada, atividade física moderada e vigorosa e fatores associados em estudantes do primeiro ano de uma instituição de ensino superior. Motricidade. 2014; 10(4): 16-26.

19. Plotnkoff RC, Hotz SB, Birkett NJ, Courneya KS. Exercise and the transtheoretical model: a longitudinal test of a population sample. Prev Med. 2001; 33(5): 441-52.

20. Cordeiro PC, Claumann GS, Pereira EF, Guimarães ACA, Pelegrini A. Estágios de mudança de comportamento relacionados à atividade física em acadêmicos de Educação Física. Rev Bras Ativ Fís Saúde. 2014; 19(4): 484-93.

21. Silva DAS, Pereira IMM. Estágios de mudança de comportamento para atividade física e fatores associados em acadêmicos de educação física. Rev Bras Ativ Fís Saúde. 2010; 15(1): 15-20.

22. Madureira AS, Corseuil HX, Pelegrini A, Petroski E. Associação entre estágios de mudança de comportamento relacionados à atividade física e estado nutricional em universitários. Cader Saú Púb. 2009; 25(10): 2139-46.

23. Martins MO, Petroski EL. Mensuração da percepção de barreiras para a prática de atividades físicas: uma proposta de instrumento. Rev Bras Cineantropom Desempenho Hum. 2000; 2(1): 58-65.

24. Jesus GM, Jesus EFA. Nível de atividade física e barreiras percebidas para a prática de atividades físicas entre policiais militares. Rev Bras Ciênc Esporte. 2012; 34(2): 433-48.

25. Pinheiro KC, Silva DAS, Petroski EL. Barreiras percebidas para a prática de musculação em adultos desistentes da modalidade. Rev Bras Ativ Fís Saúde. 2010; 15(3): 157-62.

26. Boscatto EC, Duarte MFS, Gomes MA. Estágios de mudança de comportamento e barreiras para a atividade física em obesos mórbidos. Rev Bras Cineantropom. Desempenho Hum. 2011; 13(5): 329-34.

27. Reichert FF, Barros AJD, Domingues MR, Hallal PC. The role of perceived personal barriers to engagement in leisure-time physical activity. Am J Public Health. 2007; 97(3): 515-9.

28. Mauro M, Taylor V, Wharton S, Sharma A. Barriers to obesity treatment. Eur J Intern Med. 2008; 19(3): 173-80.

29. Fitzgerald A, Fitzgerald N, Aherne C. Do peers matter? A review of peer and/or friends' influence on physical activity among American adolescents. J Adolesc. 2012; 35(4): 941-58.

30. Ball K, Bauman A, Leslie E, Owen N. Perceived environmental aesthetics and convenience and company are associated with walking for exercise among Australian adults. Prev Med. 2001; 33(5): 434-40.

Endereço para

Correspondência

Andreia Pelegrini

andreia.pelegrini@udesc.br
Centro de Ciências da Saúde e do Esporte, Universidade do Estado de Santa Catarina.

Rua Pascoal Simone, 358, Coqueiros,

Florianópolis - SC. CEP: 88080-350

Telefone: (48) 3321-8687 